\title{
38th ANNUAL SASKATCHEWAN CHRISTMAS BIRD COUNT - 1979
}

Compiled by MARY I. HOUSTON, 863 University Drive, Saskatoon, Saskatchewan, S7N 0J8.

Mild weather throughout the Christmas Bird Count period made the outings an extra pleasure this year. Counters were out in 59 localities and recorded 76 species seen on Count Day with an additional five species seen during the count period but not on count days. This is the highest species count since 1974 when 79 species were seen on Count Day with four additionals during the count period.

Two species never before recorded on the Christmas Bird Count were one American Goldfinch at

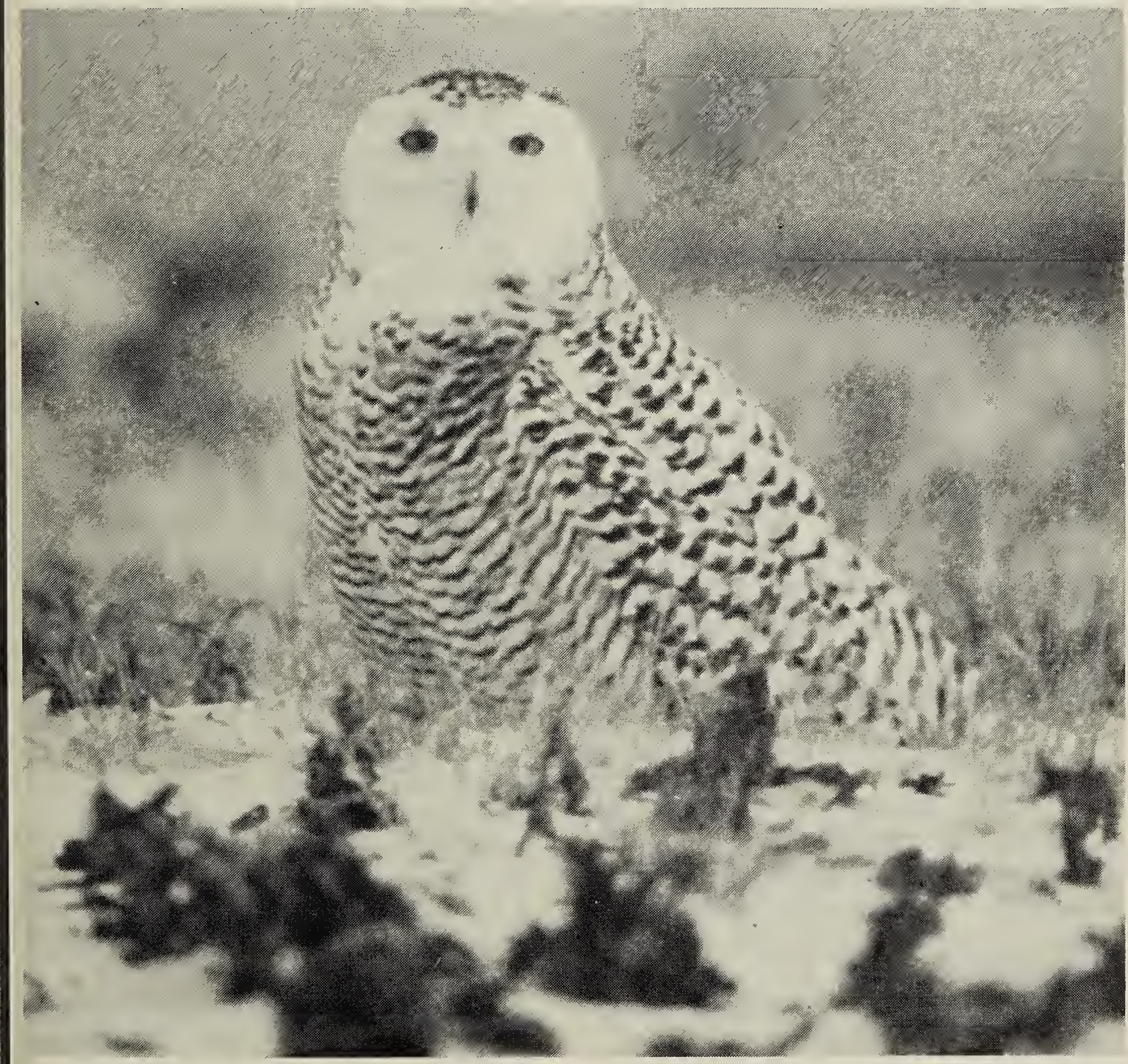

Snowy Owl. 
Saskatoon (map No. 44) and one Iceland Gull at Squaw Rapids (map No. 50); with a Cooper's Hawk at Leader (map No. 28) and a Common Snipe at Fort Walsh (map No. 15) recorded on count day rather than as additionals during count period (as previously), the 38 year Count Day total is 128 species, with two races (Oregon Junco and Audubon's Warbler) and six additional species seen during count period.

The enthusiasm of Wayne Harris and Sheila Lamont (with baby daughter Anemone along) who organized or participated in 10 counts; Guy Wapple who organized or participated in 10; and Wayne Renaud, home for Christmas holidays, who worked in nine counts,

Pintail
Goshawk
Prairie Falcon
Sage Grouse
Rock Dove
Snowy Owl
Hairy Woodpecker
Northern Three-toed Wood
Horned Lark
Blue Jay
White-breasted Nuthatch
Brown Creeper
American Robin
Mountain Bluebird
Starling
House Sparrow
Evening Grosbeak
Tree Sparrow

Table 1 lists the locations of counts and participants; Table 2 gives the distances, time and weather; Table 3 shows numbers of those species seen in more than three localities either on count day or during count period; Table 4 gives the numbers and locations of species seen in three or less localities. must be highly commended for the extensive coverage they helped to give the province.

Probably the most noticeable trend was the low number of Grosbeaks and Redpolls throughout the province. Of the 59 localities reporting, only 17 recorded Evening Grosbeaks, nine recorded Pine Grosbeaks, 12 recorded Common Redpolls and one recorded Hoary Redpolls. In all instances only small numbers of these species were seen (for example there were only 27 Pine Grosbeaks for the entire province).

Bringing up to date Renaud and Wapple's list of high individual counts published in the Blue Jay in December 1977, the following highs should be added:

$\begin{array}{rlr}4 & \text { Saskatoon } & 1979 \\ 13 & \text { Squaw Rapids } & 1979 \\ 2 & \text { Moose Jaw and } & 1977 \\ & \text { Eastend-Ravenscrag } & \\ 106 & \text { Govenlock } & 1979 \\ 2020 & \text { Saskatoon } & 1978 \\ 25 & \text { Regina } & 1979 \\ 19 & \text { Saskatoon } & 1978 \\ 8 & \text { Somme } & 1977 \\ 569 & \text { Eastend } & 1978 \\ 66 & \text { Togo } & 1977 \\ 6 & \text { Round Lake } & 1977 \\ 7 & \text { Prince Albert } & 1978 \\ 197 & \text { Saskatoon } & 1978 \\ +8 & \text { Eastend } & 1979 \\ 356 & \text { Saskatoon } & 1979 \\ 6204 & \text { Saskatoon } & 1979 \\ 292 & \text { Togo } & 1977 \\ 43 & \text { Fort Walsh } & 1979\end{array}$

Species seen during count period but not on count day are marked with $\mathrm{a}+$ before the number of individuals seen.

Information on area description for each locality and dates of species seen during count period but not on count day, are available in the files. 
TABLE 1 - Christmas Bird Count Participants

1. BATTLEFORD. Christopher Donovan, James Donovan, S. G. Sealy.

2. BIGGAR. Dale Hjertaas, Paule Hjertaas, Don Renaud, Wayne Renaud, Guy Wapple (compiler), Robert Wapple.

3. BIG GULLY CREEK. Wayne Harris (compiler), Sheila Lamont.

4. BROADVIEW. David Chaskavich, Don Weidl (compiler).

5. CLARKBORO. Muriel Carlson (compiler), Irene Gordon, Barbra Stoner.

6. DALMENY. Vi Harper, Vic Harper, Brian Sperling, Gilbert Sperling, Lorne Sperling, Lloyd Sperling (compiler).

7. DILKE. Margaret Belcher.

8. DUPEROW-RUTHILDA. Wayne Renaud, Guy Wapple (compiler).

9. EASTEND. Tom Donald, Mike Gollop (compiler), Jane Jenkins, Rick Jerema, John McIntyre, Wayne Renaud, Guy Wapple.

10. EASTEND. Mrs. Kennedy, Henri Lebastard (compiler), Mrs. McCuaig, Mrs. Phillips.

11. ELBOW. Stan Shadick, Alan R. Smith (compiler).

12. ENDEAVOUR. Norman Harris.

13. FEUDAL. Guy Wapple.

14. FORT QU'APPELLE. E. Manley Callin (compiler), Ethel Cockwill, Bernie deVries, Doug Evans, Ron Hooper, Joe Kralkay, Lois Lamontagne, Alan Mlazgar, Lorne, Don and Shaun Rowell, Gilbert Rumancik, Leo Schmalz.

15. FORT WALSH. Wayne Harris, Sheila Lamont, Wayne Renaud, Guy Wapple (compiler), Jack Wilkinson, Janet Wilkinson.

16. FRONTIER. Jack Wilkinson, Janet Wilkinson.

17. GARDINER DAM. Wayne Renaud, Guy Wapple (compiler).

18. GLAMIS-WISETON. Grev and Berna Jones.
19. GOVENLOCK. Wayne Harris (compiler), Sheila Lamont.

20. HARRIS. Wayne Renaud, Guy Wapple (compiler).

21. HUMBOLDT. Ed Brockmeyer (compiler), Clarence Saretsky, Dwayne Saretsky.

22. INDIAN HEAD. Cec and Betty Ashmore, Margaret Barclay, Peter Barrett, Vic and Margo Beaulieu, Hazel Buglãss, Com, Elizabeth, Shane and MacKinley Davidson, Irv and Richard Escott, Roger and Mavis Gray, Vic and Stella Horsman, Jim and Jean Howard, Gordon Howe, Helen King, Fred Lahrman, Ted McCurdy, Roy and Rose McLaughlin, Mrs. A. Norum, Eldon, Molly, Sonja and Wilda Norum, Lloyd and Muriel Peterson, Joan, Adam and Lorne Scott (compiler), Fred, Mary and Ken Skinner, Sandy Smith-Windsor, Ron, Donna, Andrea, Denis, and Orland Thompson, Gordon and Anne Willerth.

23. KELVINGTON. Dianne Sloan (compiler), Graham Sloan.

24. KENASTON. P. Lawrence Beckie.

25. KINDERSLEY. Jean Harris (compiler), Lane Harris, Tim Harris.

26. KUTAWAGAN LAKE (centered $19 \mathrm{~km}$ north of Semans). Wayne Harris (compiler), Sheila Lamont.

27. LAST MOUNTAIN LAKE (management unit and immediate area). Wayne Harris (compiler), Sheila Lamont.

28. LEADER. Wayne Harris (compiler), Sheila Lamont.

29. LEADER. Daisy D. Meyers.

30. LOON LAKE. Dr. P. M. Davis.

31. LUSELAND. Estelle Finley, Kerry Finley (compiler), Kim Finley, Don Martfeldt, Scott Wardley.

32. MACDOWALL. Nigel Caulkett, Dan Neves, Stan Shadick (compiler).

33. MAIDSTONE BRIDGE. Wayne Harris (compiler), Sheila Lamont, Tom Lamont. 
34. MUOSE JAW. Carl Ellis, Jeanette Fjetland, Doug Francis, Ruth Hilling, Pat Kern, Cy Knight, Leith Knight (compiler), Bernice Lewis, Moray Lewis, James McMurdo, Molly Ritchie, Jean Thomson, Wilma Young, Gus Zado.

35. NICOLLE FLATS. Dale Hjertaas (compiler), Paule Hjertaas.

36. NISBET FOREST. Rhoda Brockington, Muriel Carlson (compiler), Veitha Paine.

37. OUTLOOK. Harold Kvinge.

38. PIKE LAKE. Laurel and Kent Brace, Maddie and Bernie Gollop, Dan Mclntosh, Dan Neves, Stan Shadick (compiler), Alan and Edward Smith.

39. PRINCE ALBERT. Ansgar and Christie Aschim, Tony Capusten, B. Happ, Wayne Harris (compiler), Sheila Lamont, Margaret Lewis, Hertha McCorriston, Louis and Joice Olsen.

40. RAYMORE. Greta Harris, Wayne Harris (compiler), Sheila Lamont, Lloyd Saul.

41. REGINA. Chris Adam, Margaret Belcher, John Beveridge, Tom Beveridge, Frank Brazier, Eric Cooke, Kirsten Costain, Ann Fines, Murray Fines, Randall Fines, Jim Hines, Jim Jones, Jim Jowsey, Shirley Jowsey, Greg Kraetzig, Robert Kreba (compiler), Eric Lang, Tony Lang, Bob Luterbach, George Luterbach, Christine MacDonald, Blair McPherson, Lorraine McPherson, Brad Muir, Joe Roberts, Annika Rummenns, Bill Russan, Diane Secoy, Gary Seib, Barbara Shourounis, Glen Sutherland, Frank Switzer, Ian Switzer, Dorothy Tegart, Margaret Wigmore, Christophe Wilhelm, Pierre Wilhelm, Maureen duWors, Mary Ann Xhignesse.

42. ROUND LAKE. Doug Francis.

43. ST. WALBURG. Maurice Caulkett, Nigel Caulkett (compiler), Leonard Wourms.

44. SASKATOON. Juhachi Asai, Bob and Garth Besant, Ray Bisha, lan Creurer, Eric Diehl, Hartley Fredeen, Geoff and Muriel Galloway, Mary Gilliland,
Bernie and Madeleine Gollop, Barb Hanbidge, Bruce and John Hanbidge, Dale and Paule Hjertaas, Sam Homenuik, Dr. C. J. Houston, Stuart Houston (compiler), David Houston, Mary Houston, Ron Jensen, Blake Maybank, Jo McRobbie, Betty and Jim Mundy, Dan Neves, Bill Nickel, Jim and Pat O'Neil, Don Renaud, Wayne Renaud, John and Stan Shadick, Jim Slimmon, Alan Smith, Edward Smith, Judy Taylor, Guy Wapple.

45. SCOTT. Wayne Renaud, Guy Wapple (compiler).

46. SKULL CREEK. Eileen Bennetto, Jim Bennetto (compiler), Betty Mann, Bob Mann, Mrs. M. Mann, Marjorie Mann, Don Pearce, Michele Schuler, Ray Schuler.

47. SOMME. David Black, Donald Hooper.

48. SPIRIT LAKE. Bill and Joyce Anaka.

49. SPRING VALLEY. Allan Bogdan, Flossie Bogdan (compiler), Larry Bogdan, Nick Bogdan, Dean Goian.

50. SQUAW RAPIDS. Wayne Harris (compiler), Sheila Lamont, Don Renaud, Wayne Renaud, Guy Wapple.

51. SQUAW RAPIDS - SIPANOK CHANNEL. John Comer, Larry Prokopetz, Lyle Prokopetz.

52. WASECA. Hans deVogel, Tom Lamont, Christine Pike (compiler).

53. WASKESIU. Stan Houston, Venta Kabzems.

54. WEBB-SWIFT CURRENT. Max Mirau, Bob Peart (compiler).

55. WHITE BEAR. Darryl, Gary, Lynette, Ruby and Sig Jordheim (compiler).

56. WHITEBEECH. Ida and Lindsay Wotherspoon (compiler).

57. WOLSELEY. Dale Chay, Donald Hayward (compiler).

58. YORKTON. M. Bromley, A. Matlock, J. Matlock, Phil Pawluck (compiler).

59. WYNYARD. Charlotte Gulley, John Gulley (compiler), Rendal Kachur. 


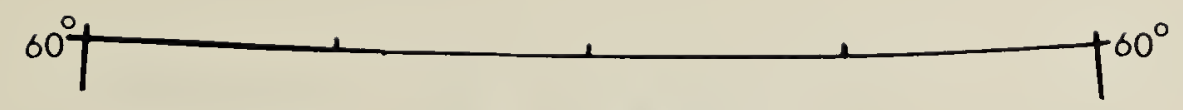

1. BATTLEFORD

2. BIGGAR

3. BIG GULLY CREEK

4. BROADVIEW

5. CLARKBORO

6. DALMENY

7. DILKE

8. DUPEROW-RUTHILDA

9. EASTEND

10. EASTEND

11. ELBOW

12. ENDEAVOUR

13. FEUDAL

14. FORT QU'APPELLE

15. FORT WALSH

16. FRONTIER

17. GARDINER DAM

18. GLAMIS-WISETON

19. GOVENLOCK

20. HARRIS

21. HUMBOLDT

22. INDIAN HEAD

23. KELVINGTON

24. KENASTON

25. KINDERSLEY

26. KUTAWAGAN LAKE

27. LAST MOUNTAIN LAKE

28. LEADER

29. LEADER

30. LOON LAKE

31. LUSELAND

32. MACDOWALL

33. MAIDSTONE BRIDGE

34. MOOSE JAW

35. NICOLLE FLATS

36. NISBET FOREST

37. OUTLOOK

38. PIKE LAKE

39. PRINCE ALBERT

40. RAYMORE

41. REGINA

42. ROUND LAKE

43. ST. WALBURG

44. SASKATOON

45. SCOTT

46. SKULL CREEK

47. SOMME

48. SPIRIT LAKE

49. SPRING VALLEY

50. SQUAW RAPIDS

51. SQUAW RAPIDS-SIPANOK CHANNEL

52. WASECA

53. WASKESIU

54. WEBB-SWIFT CURRENT

55. WHITE BEAR

56. WHITEBEECH

57. WOLSELEY

58. YORKTON

59. WYNYARD

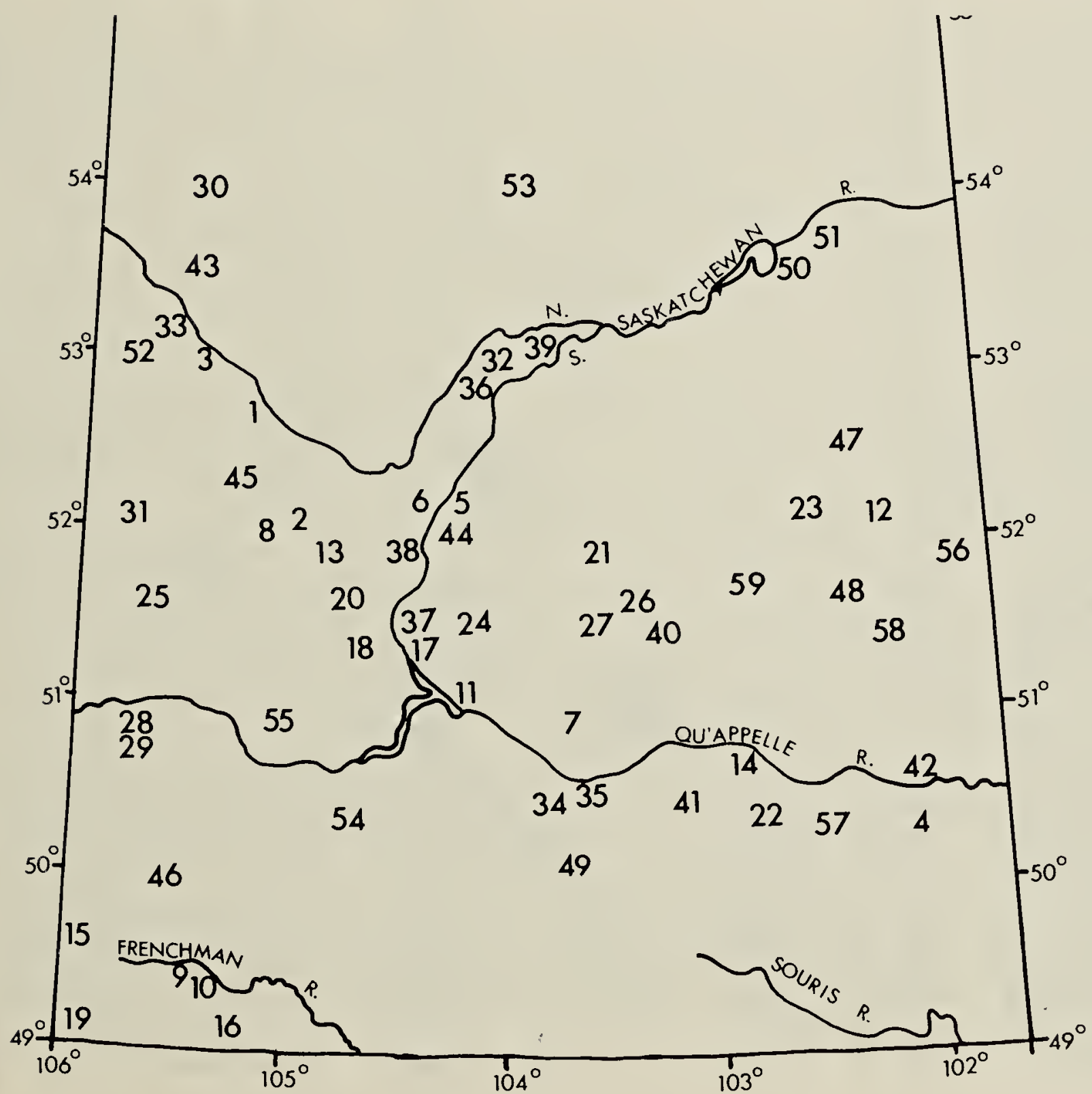




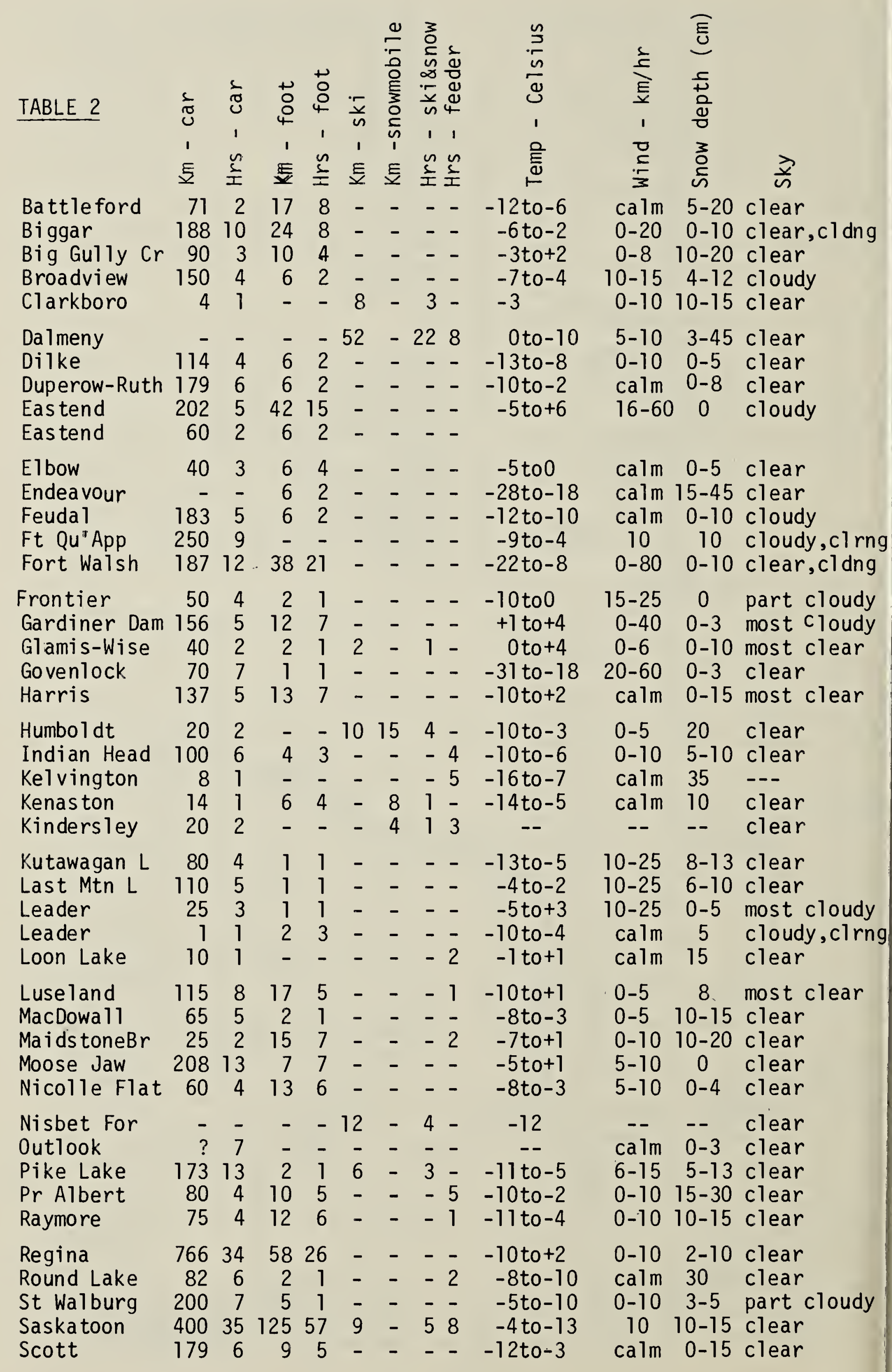




\section{TABLE 3-1}

Mallard

Com Goldeneye

Com Merganser

Goshawk

Roughleg Hawk

Golden Eagle

Bald Eagle

Prairie Falcon

Merlin

Ruffed Grouse

Sharp-t Grouse

Ring-n Pheasant

Gray Partridge

Rock Dove

G Horned OW1

Snowy Ow1

Short-ear Ow1

Com Flicker

Pileated Wdpkr

Hairy Wdpkr

Downy Wdpkr

Horned Lark

Gray Jay

Blue Jay

B-b Magpie

Com Raven

Black-c Ch'dee

Boreal Ch'dee

White-br Nuth'ch

Red-br Nuth'ch

Brown Creeper

Am Robin

Golden-c Kinglet

Bohem Waxwing

Cedar Waxwing

N Shrike

Starling

House Sparrow

Eveng Grosbeak

Pine Grosbeak

Com Redpol1

Red Crossbill

White-w Crossbill

Snow Bunting

Species in

Table 4

Count day species

Count period

species

count day indiv

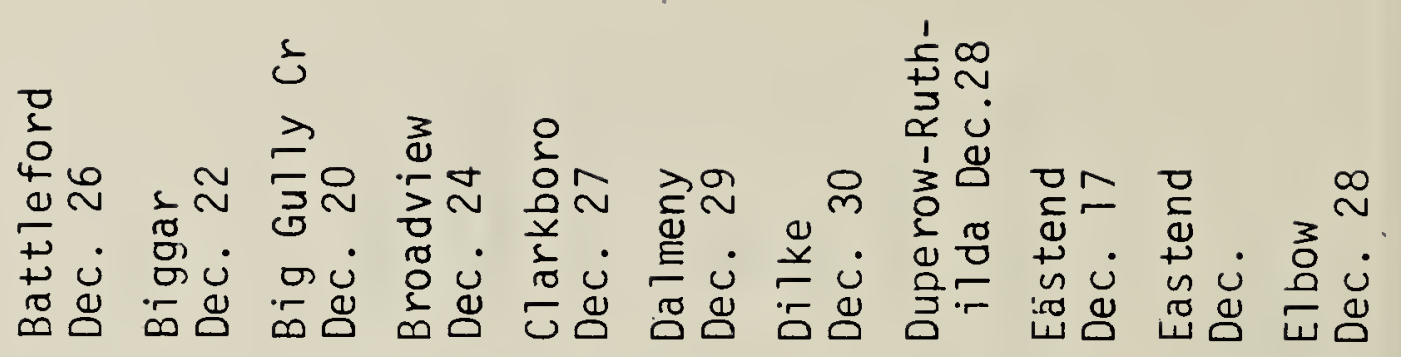
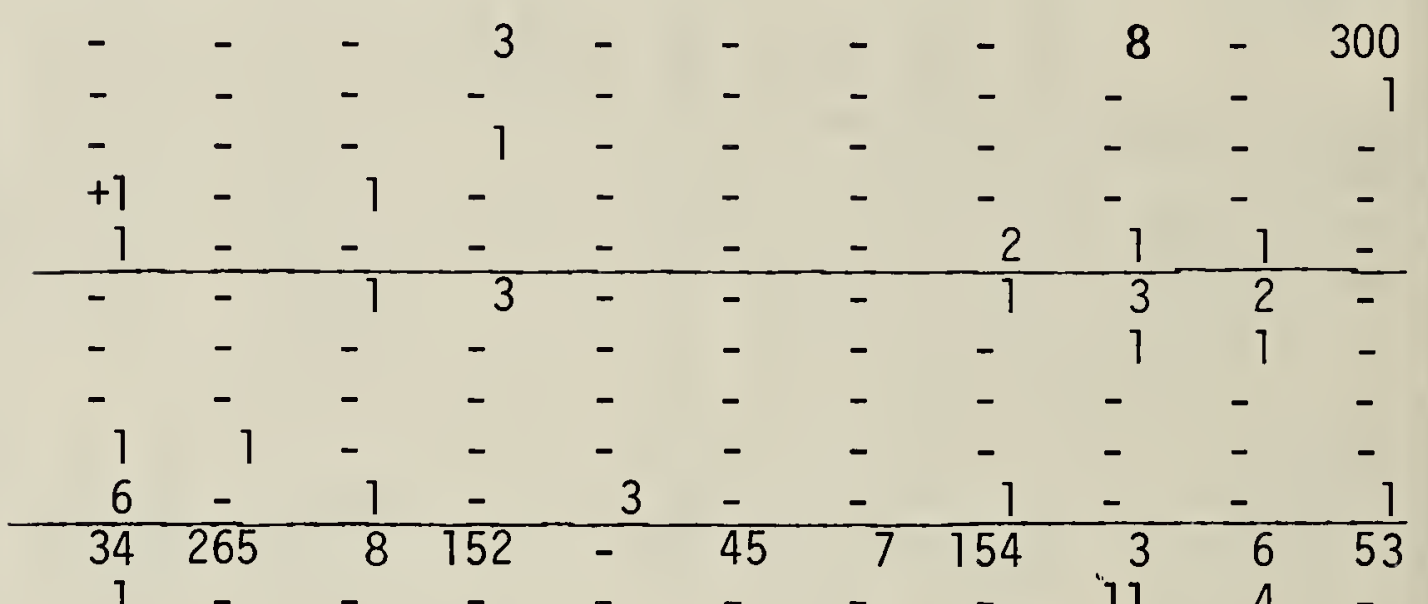

$\begin{array}{rrrrrrrrrrr}1 & - & - & - & - & - & - & - & 11 & 4 & - \\ - & 21 & - & 18 & 4 & 17 & 32 & 12 & 3 & 12 & - \\ 142 & 119 & 15 & 2 & 14 & 124 & 12 & 31 & 140 & - & 11\end{array}$

\begin{tabular}{rrrrrrrrrr}
1 & 3 & 1 & - & - & 2 & - & 1 & 3 & 1 \\
\hline- & +1 & 1 & - & - & +1 & +1 & 3 & - & 1
\end{tabular}

\begin{tabular}{rrrrrrrrrrr}
- & - & - & - & - & - & - & +1 & - & - & - \\
- & - & - & - & - & - & - & - & +1 & 2 & - \\
- & - & 1 & - & - & - & - & - & - & - & - \\
2 & - & 4 & - & 3 & 2 & - & 1 & 1 & - & - \\
\hline 3 & 1 & 1 & - & 2 & 2 & - & 1 & 1 & - & 1
\end{tabular}

$\begin{array}{rcccccccccc}+1 & - & - & - & - & - & 14 & - & 1 & 6 & 8 \\ - & - & - & - & - & - & - & - & - & - & - \\ 1 & - & 1 & - & - & 6 & - & - & 1 & 2 & -\end{array}$

\begin{tabular}{lllllllllll}
30 & 128 & 18 & 19 & 2 & 38 & 10 & 67 & 198 & 36 & 11 \\
\hline- & - & 1 & - & - & - & - & - & - & - & -
\end{tabular}

$\begin{array}{lllllllllll}15 & 10 & 14 & 10 & 12 & 13 & 4 & - & 63 & 6 & 6\end{array}$

\begin{tabular}{rcccccccccc}
- & - & - & - & - & - & - & - & - & - & - \\
- & - & - & - & - & - & - & - & - & - & - \\
- & - & 4 & - & - & - & - & - & 39 & - & - \\
\hline- & +1 & - & - & - & - & - & - & 1 & - & - \\
- & - & - & - & - & - & - & - & +1 & - & -
\end{tabular}

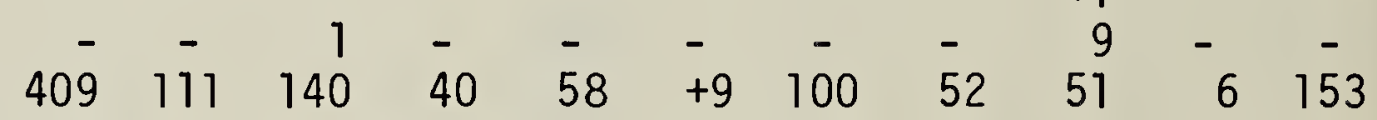

\begin{tabular}{ccccccccccc}
- & - & - & - & - & - & - & - & - & - & - \\
\hline 1 & - & - & - & - & - & - & - & +1 & - & -
\end{tabular}

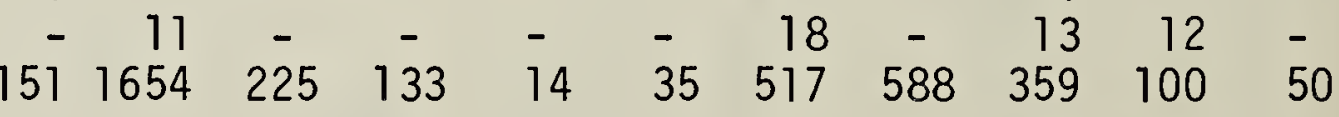

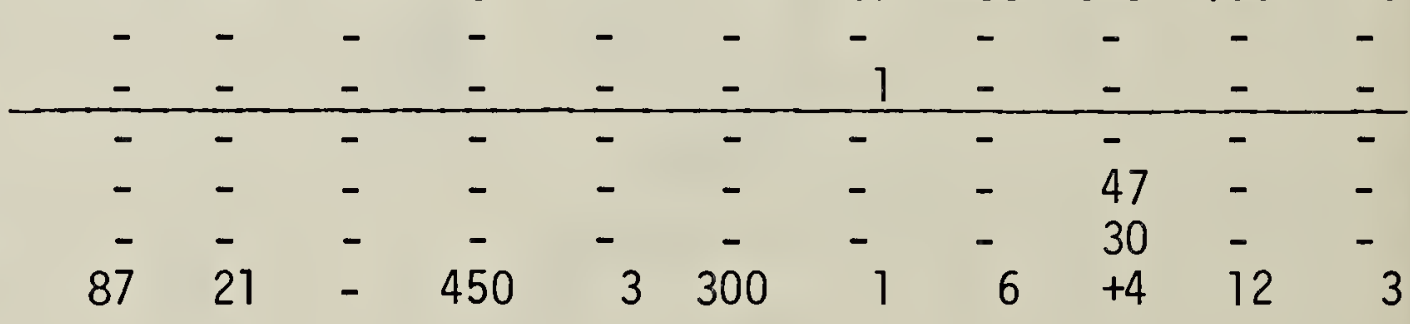

$\begin{array}{rrrrrrrrrrr}0 & 0 & 0 & 0 & 0 & 0 & 0 & 0 & 3 & 2 & 1\end{array}$

$\begin{array}{lllllllllll}16 & 12 & 18 & 11 & 10 & 11 & 11 & 14 & 26 & 18 & 14\end{array}$

$\begin{array}{rrrrrrrrrrr}18 & 14 & 18 & 11 & 10 & 13 & 12 & 15 & 30 & 19 & 14\end{array}$ $\begin{array}{lllllllllll}885 & 2345 & 438 & 831 & 115 & 584 & 716 & 920 & 997 & 212 & 600\end{array}$ 


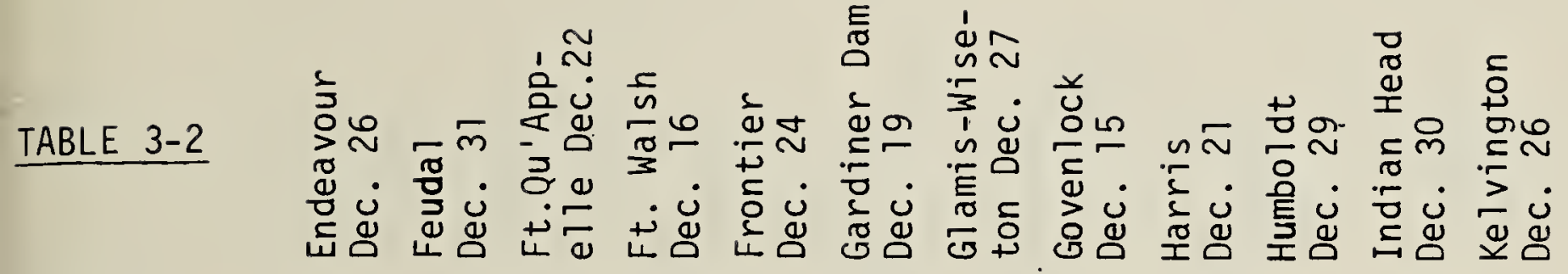

Mallard

C Goldeneye

C Merganser

Goshawk

R-leg Hawk

Golden Eagle

Bald Eagle

Prairie Falc

Merlin

Ruff Grouse

$S-t$ Grouse

R-n Pheasant

Gray Partr

Rock Dove

G H OWl

Snowy Owl

Sh-ear Owl

C Flicker

Pileat Wdpkr

Hairy Wdpkr

Downy Wdpkr

Horned Lark

Gray Jay

Blue Jay

B-b Magpie

$C$ Raven

Bl-c Ch'dee

Boreal Ch'de

Wh-br Nut

Red-br Nut

Brown Creep

Am Robin

G-c Kinglet

Boh Waxwg

Cedar Waxwg

N Shrike

Starling

House Spar

Eve Grosbk

Pine Grosbk

C Redpoll

Red Xbill

W-w Xbill

Snow Bunting

\begin{tabular}{lccccccccccc}
- & - & 120 & 4 & - & 454 & - & - & - & - & 1 & - \\
- & - & 1 & - & - & 52 & - & - & - & - & 1 & - \\
- & - & - & - & - & 3 & - & - & - & - & - & - \\
- & - & - & 1 & - & - & - & - & - & 1 & - & - \\
- & - & - & 2 & - & - & - & - & - & - & - & - \\
\hline- & - & - & 2 & 2 & 1 & - & 1 & 1 & - & - & - \\
- & - & +1 & 2 & - & 4 & - & - & - & - & 2 & - \\
- & - & - & 1 & - & 1 & - & - & - & - & - & - \\
- & 1 & - & 1 & +1 & - & - & - & - & - & +1 & - \\
- & - & 1 & 3 & - & 1 & - & - & - & - & 10 & - \\
\hline 13 & 18 & 51 & 11 & 7 & - & 4 & - & 303 & 25 & 20 & 7 \\
- & - & +2 & 10 & 19 & 2 & - & 1 & - & - & 1 & -
\end{tabular}

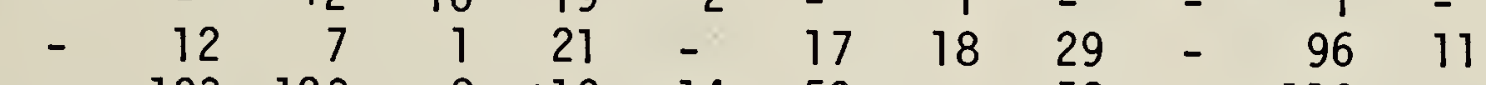

- $\begin{array}{rllllll}103 & 123 & 8 & \end{array}$

Species in

Table 4

Ct day sp

Ct perd $s p$

$-\frac{1}{-}+1$

$C t$ indiv
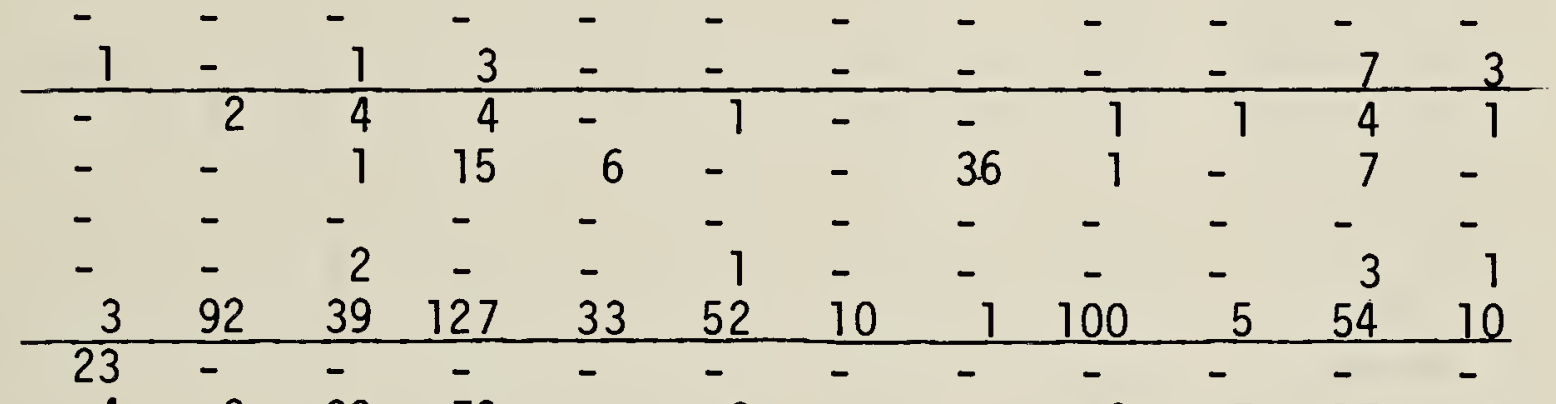

$\begin{array}{ccccccccccccc}4 & 2 & 20 & 79 & - & 8 & - & - & 8 & 7 & 17 & 4\end{array}$

\begin{tabular}{llllllllllll}
- & - & - & - & - & - & - & - & - & - & - & - \\
- & - & +2 & - & - & - & - & - & - & - & - & - \\
- & - & - & 27 & - & - & - & - & - & - & 3 & - \\
\hline & - & - & 2 & - & - & - & - & - & - & - & -
\end{tabular}

\begin{tabular}{cccccccccccc}
- & - & +1 & - & - & 1 & - & - & - & - & - & - \\
- & - & - & 37 & - & - & - & - & - & - & - & - \\
- & 265 & 50 & 56 & +22 & 110 & - & - & 48 & 1 & 62 & - \\
- & - & - & - & - & - & - & - & - & - & - & - \\
\hline- & - & 2 & 1 & - & - & - & - & - & - & +1 & -
\end{tabular}

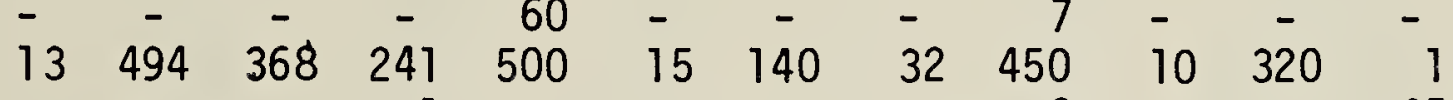

\begin{tabular}{rcccccccccc}
- & - & - & 1 & - & - & - & - & 6 & - & - \\
- & - & - & - & - & - & - & - & - & - & - \\
\hline
\end{tabular}




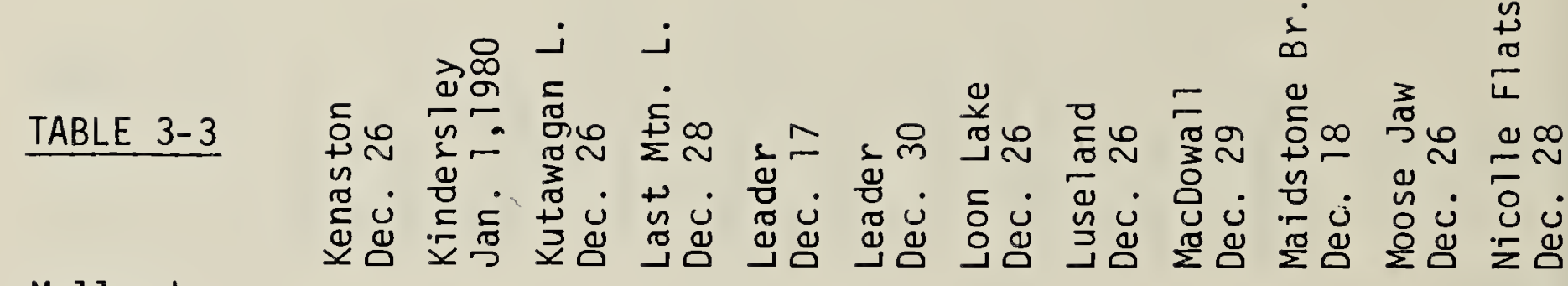

Mallard

C Goldeneye

C Merganser

Goshawk

R-leg Hawk

Golden Eagle

Bald Eagle

Prairie Falc

Merlin

Ruff Grouse

S- $t$ Grouse

R-n Pheasant

Gray Partr

Rock Dove

G H OWT

Snowy Ow1

Sh-ear Ow1

C Flicker

Pileat Wkpkr

Hairy Wdpkr

Downy Wkpkr

Horned Lark

Gray Jay

Blue Jay

B-b Magpie

C Raven

B1-C Ch' dee

Boreal $\mathrm{Ch}^{\prime}$ de

Wh-br Nut

Red-br Nut

Brown Creep

Am Robin

G-c Kinglet

Boh Waxwg

Cedar Waxwg

$N$ Shrike

Starling

House Spar

Eve Grosbk

Pine Grosbk

C Redpoll

Red Xbill

W-w Xbil1

Snow Bunting

\begin{tabular}{cccccccccccc}
- & - & - & - & - & - & - & - & - & - & - & - \\
- & - & - & - & - & - & - & 1 & - & - & 1 & - \\
- & - & - & - & - & - & +2 & - & - & 11 & - & 1 \\
\hline 25 & - & 8 & - & 1 & 11 & - & 18 & - & 3 & - & 26 \\
- & - & 1 & +1 & 1 & 6 & - & - & - & - & 12 & -
\end{tabular}

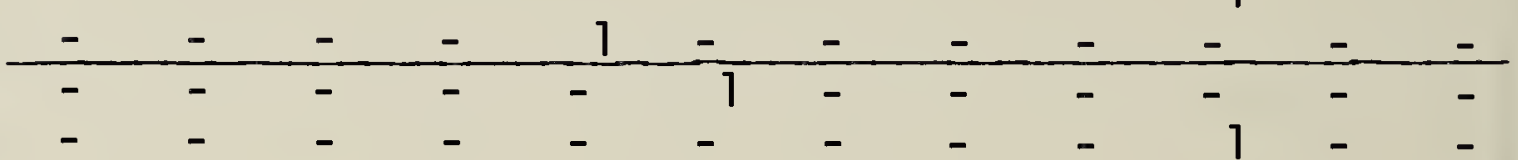

\begin{tabular}{rrrrrrrrrrrr}
16 & - & 45 & 55 & 18 & - & - & 3 & - & - & 32 & - \\
3 & 3 & 16 & 2 & 23 & - & - & 29 & - & - & 237 & 9 \\
1 & - & 2 & 3 & - & - & - & - & - & 3 & - & - \\
\hline
\end{tabular}

Species in

Table 4

Ct day sp

$c t$ perd $s p$

$c t$ indiv
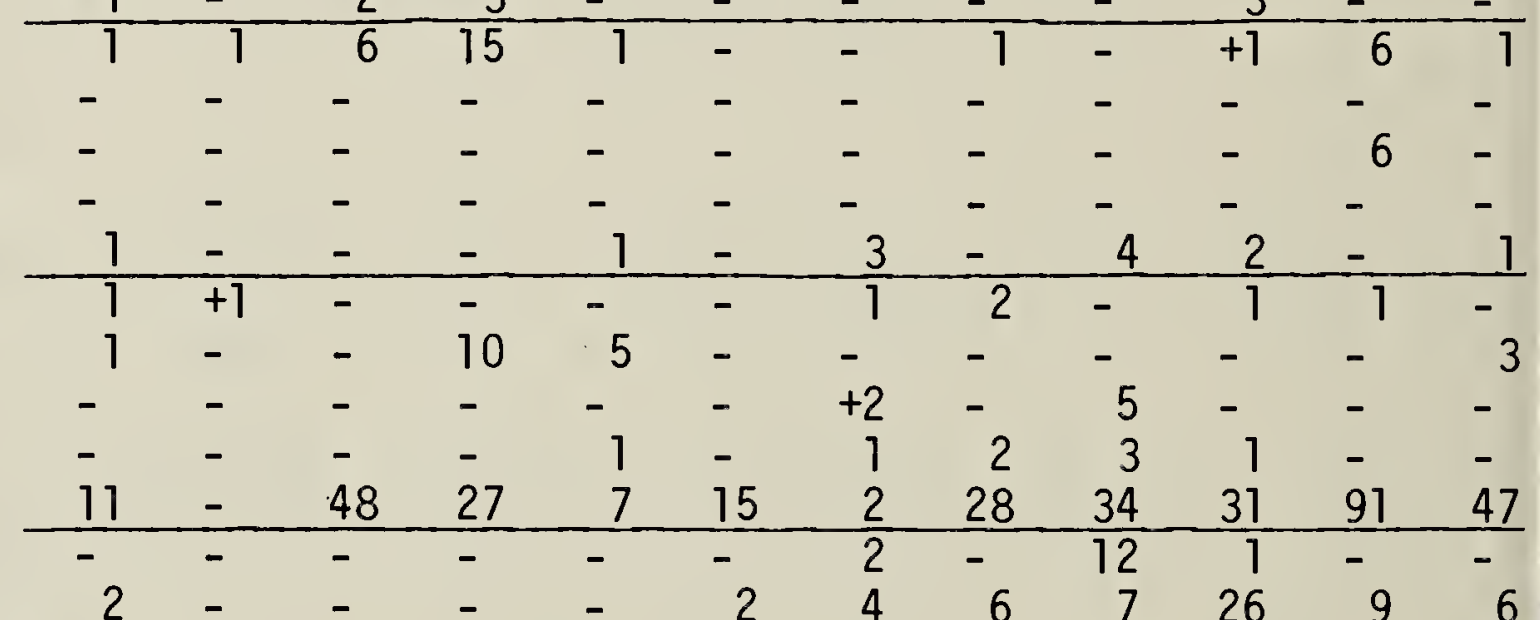

$\begin{array}{lllllllllllll}2 & - & - & - & - & - & - & - & 10 & - & -\end{array}$

\begin{tabular}{cccccccccccc}
- & - & - & - & - & - & - & - & - & - & - & - \\
- & - & - & - & - & - & - & - & - & - & - & - \\
\hline- & - & - & - & - & - & - & - & 2 & - & +1 & -
\end{tabular}

$\begin{array}{llllllllllll}- & - & - & - & - & - & - & - & - & - & +1 & - \\ - & - & - & - & - & - & - & - & - & - & - & -\end{array}$

\begin{tabular}{|c|c|c|c|c|c|c|c|c|c|c|}
\hline+7 & +20 & - & - & - & - & 6 & 256 & 38 & 2 & 1 \\
\hline- & - & - & - & - & - & - & 7 & - & $=$ & 30 \\
\hline$\overline{-}$ & $=$ & -1 & - & - & 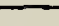 & 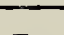 & & & & - \\
\hline
\end{tabular}

$\begin{array}{llllllllllll}- & - & - & - & - & - & - & - & - & +1 & - & - \\ - & - & 3 & - & 9 & - & - & 3 & - & - & 51 & 1\end{array}$

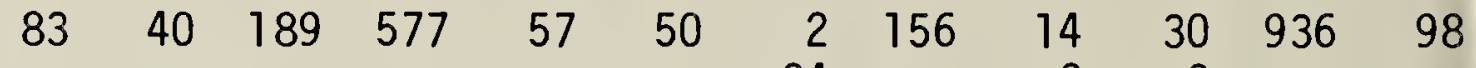

\begin{tabular}{rrrrrrrrrrrr}
- & - & - & - & - & - & 24 & - & 2 & 2 & - & - \\
- & - & - & - & - & - & - & - & 7 & 3 & - & - \\
\hline- & - & - & - & - & - & - & 4 & $33+17$ & - & -
\end{tabular}

$-\quad-\quad-\quad-$

$63+50-67 \quad 1-+50+250 \quad 13 \quad 11$




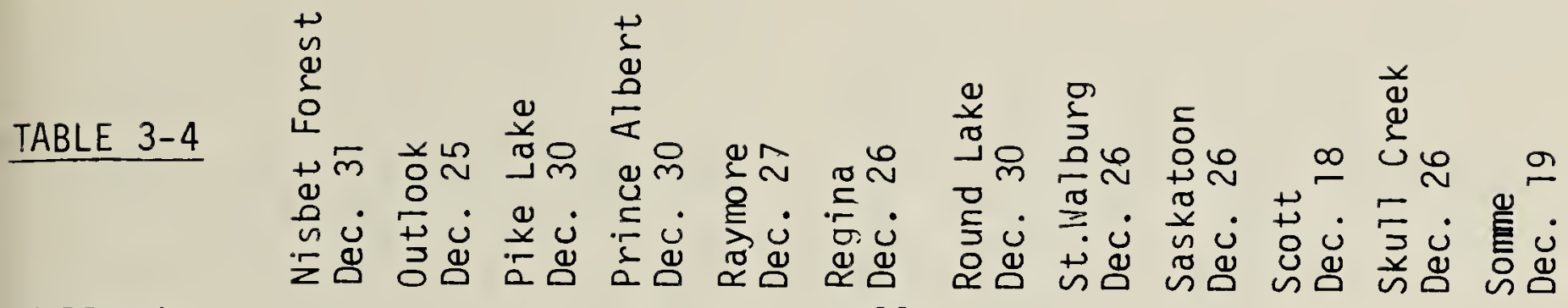

Mallard

C Goldeneye

C Merganser

Goshawk

R-leg Hawk

Golden Eagle

Bald Eagle

Prairie Falc

Merlin

Ruff Grouse

S-t Grouse

R-n Pheasant

Gray Partr

Rock Dove

G H OWT

Snowy Owl

Sh-ear Ow1

C Flicker

Pileat Wdpkr

Hairy Wdpkr

Downy Wdpkr

Horned Lark

Gray Jay

Blue Jay

B-b Magpie

C Raven

Bl-c Ch'dee

Boreal $\mathrm{Ch}^{\prime}$ de

Wh-br Nut

Red-br Nut

Brown Creep

Am Robin

G-c Kinglet

Boh Waxwg

Cedar Waxwg

N Shrike

Starling

House Spar

Eve Grosbk

Pine Grosbk

C Redpoll

Red Xbill

W-W Xbill

Snow Bunting

Species in

Table 4

Ct day $\mathrm{sp}$

Ct perd sp

$c t$ indiv

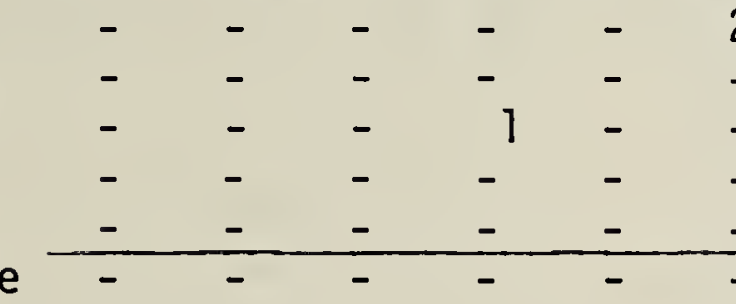

$\begin{array}{llllllllllll}- & - & - & - & - & - & +2 & - & - & - & 1 & - \\ - & - & - & - & - & - & - & - & - & - & +1 & -\end{array}$

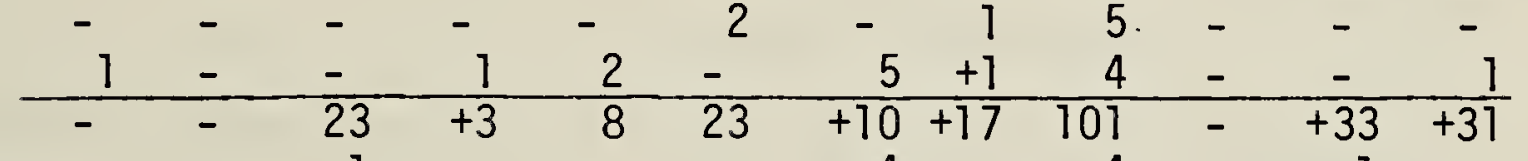

- -1 - 1 - -4 - 4 - $4-1$ -

- $-2-2311825-10365-1$

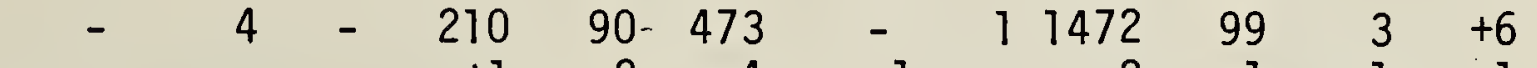

$\begin{array}{llrrrrrrrrrr}- & - & - & +1 & 2 & 4 & 1 & - & 2 & 1 & 1 & 1 \\ - & 1 & 2 & +1 & 4 & 25 & - & - & 2 & 2 & +1 & -\end{array}$

$\begin{array}{llllllllllll}- & - & - & - & - & 3 & - & - & 2 & - & - & - \\ - & - & - & - & - & - & - & - & - & - & - & 1\end{array}$

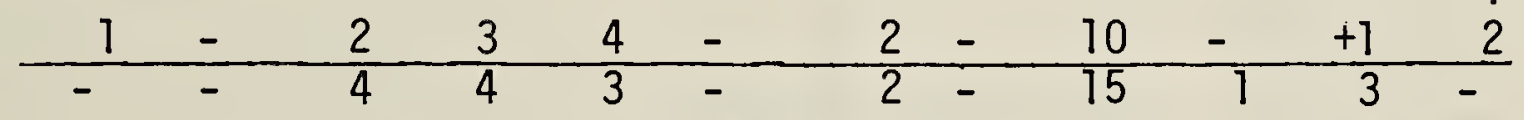

$\begin{array}{lllllllllll} & - & - & - & +5 & 6 & - & - & - & - & 1\end{array}$

$\begin{array}{rrrrrrrrrrrr}1 & 1 & - & +2 & - & - & - & 1 & - & - & - & 1 \\ - & +1 & 11 & 4 & 1 & - & +1 & 1 & 33 & - & - & 4\end{array}$

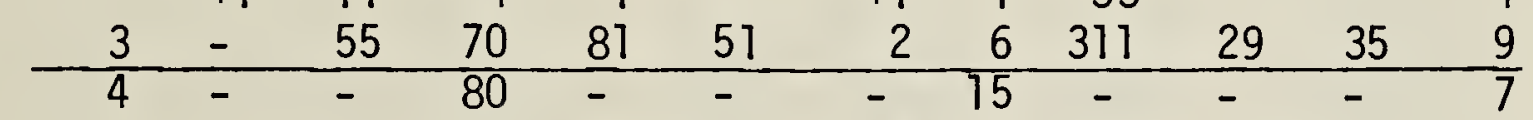

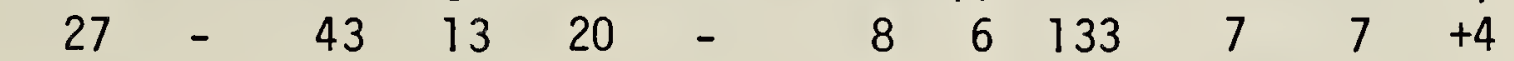

- - - $8-5-1-1-7$

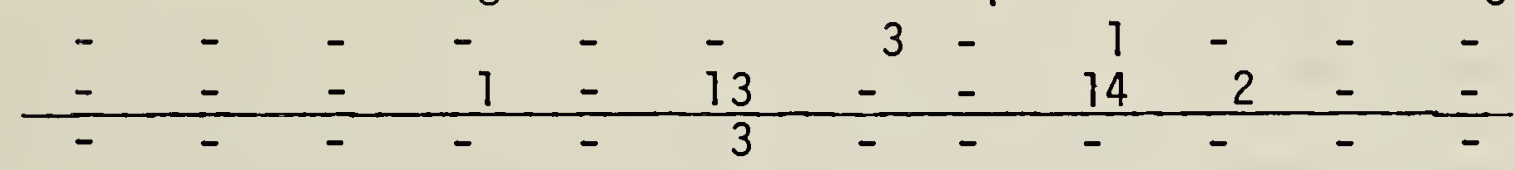

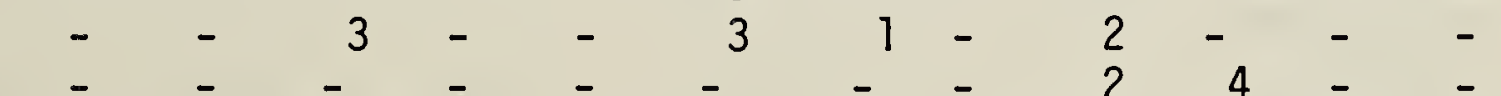

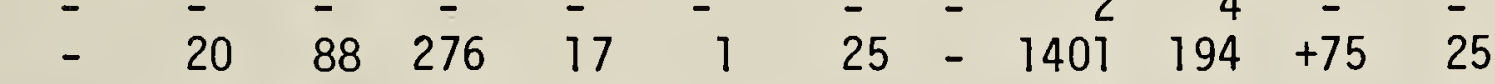

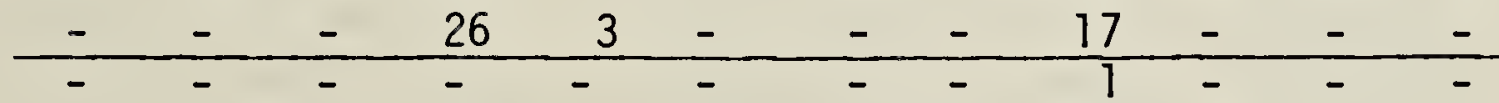

- $\quad-\quad-36-13-10-35618-10$

$\begin{array}{llllllllllll}- & 10 & 121 & 511 & 862 & 2121 & 25 & 10 & 6204 & 346 & 83 & 47\end{array}$

- $-2126-5-4-8$ - $\quad$ - 19

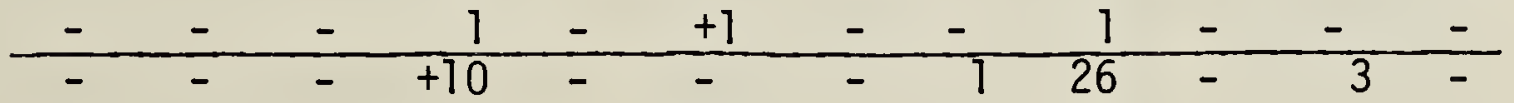

$\begin{array}{llllllllllll}- & - & - & - & - & 15 & - & 4 & 31 & - & - & - \\ - & - & - & 2 & - & - & - & - & 1 & - & - & 12\end{array}$

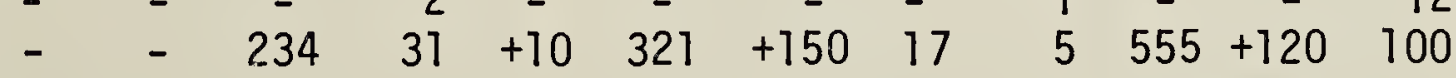

$\begin{array}{rrrrrrrrrrrr}0 & 0 & 0 & 0 & 1 & 2 & 0 & 0 & 9 & 0 & 1 & 2 \\ 6 & 4 & 14 & 19 & 14 & 21 & 14 & 12 & 41 & 15 & 11 & 16 \\ 6 & 6 & 14 & 24 & 17 & 22 & 18 & 14 & 42 & 15 & 18 & 22 \\ 37 & 27 & 591 & 1404 & 1120 & 4379 & 108 & 6410381 & 1325 & 144 & 235\end{array}$




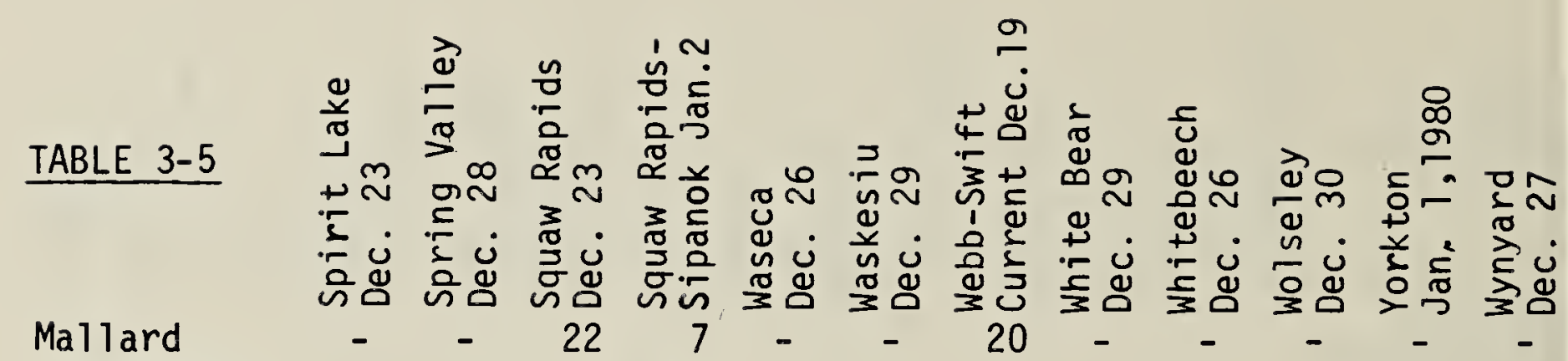

C Goldeneye

C Merganser

- $\quad-15042$

Goshawk

$-\quad-$

R-leg Hawk

Golden Eagle

$+1-\begin{array}{llll}4 & - & -\end{array}$

$+1+1$

Bald Eagle

Prairie Falc

Merlin

Ruff Grouse

S- $t$ Grouse

R-n Pheasant

Gray Partr

Rock Dove

G H OWl

Snowy Ow1

Sh-ear Owl

C Flicker

Pileat Wdpkr

Hairy Wdpkr

Downy Wdpkr

Horned Lark

Gray Jay

B1 ue Jay

B-b Magpie

C Raven

B1-C Ch'dee

Boreal $\mathrm{Ch}^{\prime} \mathrm{de}$

Wh-br Nut

Red-br Nut

Brown Creep

Am Robin

G-c Kinglet

Boh Waxwg

Cedar Waxwg

N Shrike

Starling

House Spar

Eve Grosbk

Pine Grosbk

C Redpol1

Red Xbill

W-w Xbil1

Snow Bunting

\begin{tabular}{ccc}
+ & - & - \\
- & 1 & - \\
- & - & 1 \\
- & +1 & - \\
- & +4 & - \\
7 & - & \\
\hline 1 & 49 & 1
\end{tabular}

$+326-+10-$

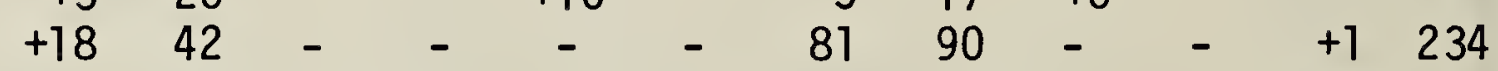

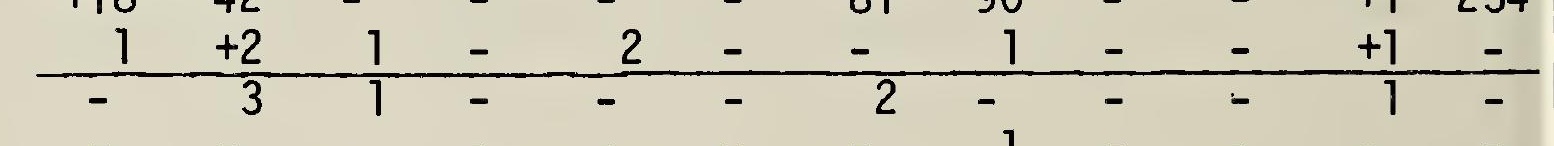

Species in

Table 4

Ct day $s p$

Ct perd $\mathrm{sp}$

$c t$ indiv

$+2$

\begin{tabular}{rrrrr}
+2 & - & 3 & - & - \\
6 & - & 18 & - & 2 \\
\hline 7 & - & 2 & 1 & +1
\end{tabular}

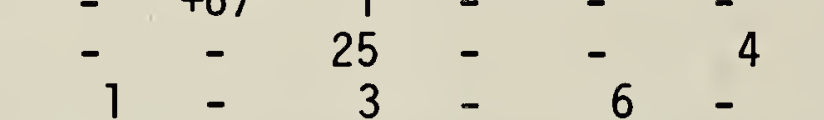

\begin{tabular}{rrrrrrrrrrrr}
24 & 16 & 8 & 1 & 6 & - & 43 & 23 & +4 & 4 & 4 & 10 \\
\hline+1 & - & 148 & 27 & 1 & 5 & - & - & 6 & - & - & -
\end{tabular}

$24-24 \quad 2128$ -

$1-$

$\begin{array}{cccc}- & - & - & - \\ - & - & 2 & -\end{array}$

$\begin{array}{llll}- & - & 6 & -\end{array}$

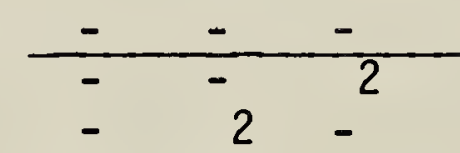

120800

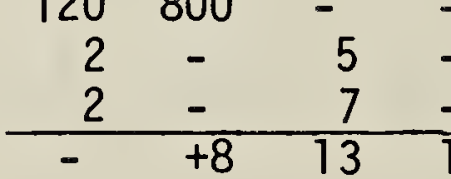

$-3$

$\begin{array}{rrrrrrrrrrrr}0 & 1 & 11 & 2 & 0 & 1 & 1 & 0 & 0 & 1 & 0 & 0 \\ 14 & 10 & 35 & 13 & 10 & 4 & 10 & 10 & 5 & 8 & 5 & 9 \\ 19 & 16 & 35 & 13 & 15 & 5 & 13 & 10 & 13 & 8 & 10 & 10 \\ 200 & 1079 & 538 & 103 & 949 & 18 & 487 & 305 & 46 & 131 & 311 & 496\end{array}$


TABLE 4 - Species Seen in Three or Fewer Localities

Western Grebe: Gardiner Dam, 1.

Canada Goose: Gardiner Dam, 28; Regina, 1160; Saskatoon, 1.

Snow Goose: Saskatoon, 1.

Gadwall: Gardiner Dam, 1.

Pintail: Saskatoon, 4; Squaw Rapids, 1.

American Wigeon: Gardiner Dam, 1.

Canvasback: Gardiner Dam, 2.

Lesser Scaup: Gardiner Dam, 1; Saskatoon, 2.

Bufflehead: Gardiner Dam, 1.

Hooded Merganser: Gardiner Dam, 2.

Red-breasted Merganser: Gardiner Dam, 1.

Duck Spp: Eastend (map No. 10), 2.

Sharp-shinned Hawk: Fort Walsh, 1; Saskatoon, 1.

Cooper's Hawk: Leader (map no. 28), 1.

Eagle Spp: Saskatoon, 1; Wolseley, 1.

Gyrfalcon: Elbow, 1; Squaw Rapids, 1.

Spruce Grouse: Squaw Rapids, 1; Squaw

Rapids-Sipanok Channel, 1;

Waskesiu, 1.

Sage Grouse: Fort Walsh, +2; Govenlock, 106.

Common Snipe: Fort Walsh, 1.

Glaucous Gull: Gardiner Dam, 3.

Iceland Gull: Squaw Rapids, 1.

Hawk Owl: Squaw Rapids, 1; Squaw Rapids-Sipanok Channel, 2.

Great Gray Owl: Squaw Rapids, 1.

Boreal Owl: Maidstone Bridge, +1 .

Saw-whet Owl: Webb-Swift Current, +1 .

Owl Spp: Squaw Rapids, 1.

Black-backed Three-toed Woodpecker:

Somme, +1; Squaw Rapids, 2.

Northern Three-toed Woodpecker: Fort

Walsh, 1; Somme, 2; Squaw Rapids, 1.

Woodpecker Spp: Fort Walsh, 3; Squaw Rapids, 5.

Common Crow: Moose Jaw, 3.
Mountain Bluebird: Eastend (map No. 10), +8 .

Townsend Solitaire: Fort Walsh, +1 .

Redwinged Blackbird: Regina, 1.

Rusty Blackbird: Fort Walsh, 1; Spring Valley, +1

Blackbird Spp: Eastend (map No. 9), 7.

Gray-crowned Rosy Finch: Fort Walsh, 22.

Hoary Redpolt Maidstone Bridge, +2 .

Redpoll Spp: Fort Walsh, 2; Raymore, +1; Squaw Rapids, 191.

Pine Siskin: Saskatoon, 5.

American Goldfinch: Saskatoon, 1.

Dark-eyed Junco: Eastend (map No. 9), 1; Fort Walsh, 3.

Tree Sparrow: Eastend (map No. 9), 2; Fort Walsh, 43; Skull Creek, 6.

Harris' Sparrow: Saskatoon, 1.

\section{TOGO, Dec. 20}

Again interested people with bird feeders within a $11.5 \mathrm{~km}$ radius of Togo shared the 3,000 Ibs. of sunflower seeds brought from southern Manitoba by Cusie May, and carefully recorded all observations at their feeders from $20 \mathrm{Dec}$. to 1 Jan. The best day was 20 Dec. The following species were recorded: Sharp-tailed grouse, 51; Hairy Woodpecker, 2; Downy Woodpecker, 2; Blue Jay, 1; Black-billed Magpie, 10; Common Raven, 12; Blackcapped Chickadee, 29; Boreal Chickadee, 3; Bohemian Waxwing, 58; House Sparrow, 91; Evening Grosbeak, 144; Pine Siskin, 6. Additionals seen during the count period included Ruffed Grouse, 8, Dec. 27; Gray Partridge, 9, Dec. 25; Common Redpoll, 2, Dec. 21; Darkeyed Junco, 3, Dec. 25. Observers were: Bill and Gwen Appel, Bert and Doris Franklin, Phil and Jean Hern, Vic and OIga Hilderman, Bill and Vi 
Holiday, Walter and Ethel Krupp, Albert and Tillie Lowenberger, Cusie and Wanda May (compiler), Dick and Mary Smith, Howard and Donelda Wilson, Orville and Elaine Wilson.

We regret this count was too late to enter onto the charts, but are very pleased to have received it so the information is recorded for future use.

FORT SMITH, NORTHWEST TERRITORIES

DATE: 26 December, 1979.

WEATHER: Overcast with sunny patches; temperature minus one degree celsius, establishing a new high for the area, previous record was minus six degrees celsius in 1959; snow depth approximately 25 $\mathrm{cm}$; daylight hours $0900 \mathrm{hrs}$. to 1600 hrs.

ROUTES COVERED: Fort Smith west along NWT Highway 5 to the Foxhole Road intersection; Fort Smith south along the Pine Lake Road to Salt River; Fort Smith east along the Hay Camp Road to Fort Fitzgerald. 150 $\mathrm{km}$ in $3.5 \mathrm{hrs}$.

BIRDS SIGHTED: Common Raven, 175; Gray Jay, 22; Redpoll sp., 93; Downy Woodpecker, 2; Hairy Woodpecker, 2; Boreal Chickadee, 13; Black-Capped Chickadee, 14; Pine Grosbeak, 26; Pine Siskin, 20; White-Winged Crossbill, 16; House Sparrow, 2; Goshawk, 1; Dark-Eyed Junco, 2; Bohemian Waxwing, 1. Add Ruffed Grouse, 1; Spruce Grouse, 1; December 27.

SUMMARY: 16 species, 391 individuals (new record!).

CONTRIBUTORS: Ian Church, Nancy Church; Marylyn Anions; Jueneva

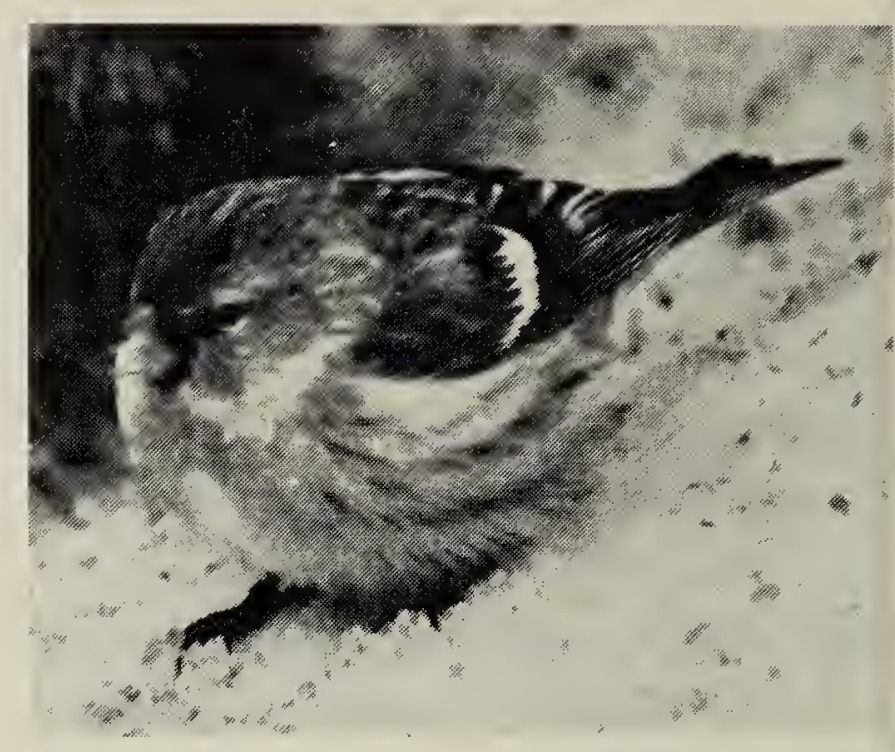

Common Redpoll.

Fred W. Lahrman

Green, Jaida Green; Gisele Samoila, Dwight Krause; Don Brannigan, Isabel Brannigan and Tommy Brannigan; Harold Pankratz, Barb Pankratz; Dr. Martha Jalkotzy; Bernie Lieff; Dan Graham (compiler) and Linda Graham.

\section{YELLOWKNIFE, NORTHWEST TERRITORIES}

23 December 1979; $139 \mathrm{~km}$ by car and $30 \mathrm{~km}$ on foot in 21.6 party hours; temperature $-22^{\circ} \mathrm{C}$; wind nil; overcast, with snow showers in morning; 4 species, 577 individuals.

Willow Ptarmigan, 51; Gray Jay, 3; Common Raven 493; House Sparrow, 30. ADD: Rock Dove, 3, 28 December.

JoAnne Allison, Wayne Bryant; Tom Dafoe; Doug and Sandra Desjardins; Hannah Diamond; Bob and Kuluk Gamble; Carol, Troy, and George Gibson; Jonni Graves; Lee and Andrew Harding; Doug Heard; Don Karasiuk (compiler), Kathy Southworth; John Stephenson; Wendy Thompson. 